\title{
A New Certificateless Signcryption Scheme for Securing Internet of Vehicles
}

Beibei Cui ( $\nabla$ cuibei3@163.com )

Anhui University Longhe Campus: Anhui University

Lu Wei

Anhui University Longhe Campus: Anhui University

Wei He

Anhui Water conservancy Technical college

\section{Research Article}

Keywords: Elliptic Curve, Certificateless signcryption, Pseudonym, Timestamp mechanism

Posted Date: January 28th, 2022

DOI: https://doi.org/10.21203/rs.3.rs-1272183/v1

License: (1) This work is licensed under a Creative Commons Attribution 4.0 International License.

Read Full License 


\title{
A New Certificateless Signcryption Scheme for Securing Internet of Vehicles
}

First Beibei Cui ${ }^{1,2^{*}}$, Second Lu Wei ${ }^{2,3 \dagger}$ and Third Wei $\mathrm{He}^{3,4 \dagger}$

$1^{*}$ Department of Electronic Information ,Huishang Vocational College, Zipeng Road, Hefei, 230039, Anhui, China.

${ }^{2}$ The Anhui Engineering Laboratory of IoT Security Technologies, Anhui University, JiuLong Road, Hefei, 230039, Anhui, China.

${ }^{3}$ School of Computer Science and Technology, Anhui University, JiuLong Road, Hefei, 230039, Anhui, China.

${ }^{4}$ School of mechanical and automotive engineering, Anhui Water Conservancy Technical College, No. 18, Hema road, Feidong County, Hefei City, Anhui Province, Hefei, 231603, Anhui, China.

\author{
*Corresponding author(s). E-mail(s): cuibei3@163.com; \\ Contributing authors: \\ Great $_{h} e @ 163 . c o m ;$ dreamer $w$ eilu@163.com; \\ $\dagger$ These authors contributed equally to this work.
}

\begin{abstract}
The application of digital signature technology on the Internet of Vehicles (IoV) is affected by its network and communication environment, which requires low transmission delay, power consumption, and highsecurity requirement. To the best of our knowledge, a well-designed solution that uses signcryption technology has not been proposed in the IoV research area. Motivated by the fact, a certificateless signcryption scheme based on Elliptic Curve Digital Signature Algorithm, which also considers pseudonym and timestamp mechanism, has been designed in this paper. We prove that our proposed scheme can be reduced to solving the difficulty of the Computational Diffie-Hellman problem under the standard model, show that the scheme meets both security and efficiency requirements, and provides a comparative analysis with the state-of-the-art schemes in terms of security analysis, computational cost, and communication cost, demonstrating that our proposed scheme is suitable to be deployed in the IoV environment.
\end{abstract}


Keywords: Elliptic Curve; Certificateless signcryption; Pseudonym;

Timestamp mechanism

\section{Introduction}

In the $5 \mathrm{~g}$ era, Internet of vehicles (IoV) has developed rapidly. To meet the needs of research and application, IoV can be divided into vehicle to vehicle $(\mathrm{V} 2 \mathrm{~V})$, vehicle to infrastructure (V2I), vehicle to pedestrian (V2P), and vehicle and network(V2N) communication. They are exchange data using wired or wireless communication and store the data in the cloud environment[1].

There are security problems such as counterfeiting, tampering, and forgery[2] in all links of IoV. Anonymous authentication is the key factor to solve the problem of information security and privacy protection, it is a research hotspot in recent years. Kamat et al.[3] proposed a security framework for VANETs based on Identity-Based Cryptography (IBC). IBC was first proposed by Shamir [4] as early as 1984, the idea of an identity-based cryptosystem in which arbitrary strings can act as public keys. For example, Zhang et al.[5] proposed to use fingerprint information as identity authentication. Cui et al.[17] applied the privacy protection of edge computing used in VANETs. Raya et al.[6] proposed a conditional anonymity scheme, which requires a thirdparty trusted organization to store the correspondence between all vehicles and anonymous certificates. When the authority is not authorized, it may deliberately disclose vehicle privacy information, forge and tamper with legal vehicle identity. To solve this problem, Tzeng et al.[7] introduces the identity-based public-key cryptosystem into the Internet of vehicles and designs an identitybased public-key cryptosystem authentication scheme. The user's private key is generated by the third-party private key generator (PKG). However, if the third-party private key generation center is dishonest or malicious, it can forge the signature of any user, which has the problem of key escrow. Therefore, Al-Riyami et al.[8] put forward the concept of key generator center(KGC), pointing out that the generation of any effective signature based on obtaining the secret value of OBU and partial keys distributed by KGC at the same time. In 2007, Liu et al.[9] proposed a certificateless signature scheme. Compared with the traditional certificate-based signature scheme, the key is no longer simply determined by CA. Shim[10] designed a new certificateless signature scheme and analyzed the security of the scheme based on Computational Diffie Hellman (CDH), but Yang et al.[11] considered that the scheme is vulnerable to malicious but passive KGC attacks. In 2020, Thumbur et al.[12] proposed a certificateless signature scheme without bilinear pairing, saying that the scheme can be deployed in source constrained IoV. Mei et al.[13] proposed a certificate-less signature aggregation scheme with conditional privacy protection based on bilinear pairing. The scheme realizes complete aggregation and can be proved to be secure under the random oracle model. Ali et al.[14] designed an identity-based message authentication scheme without bilinear 
pairing for $\mathrm{V} 2 \mathrm{~V}$ secure communication. When vehicles apply to the trusted authority (TA) for registration, the TA generated pseudonyms and keys for them to protect privacy in the communication process.

Barbosa et al.[15] proposed the definition form of certificateless signcryption (CLSC), their scheme introduced signcryption. Signcryption was originally proposed by Zheng[16] for the first time, which can transmit signature and encryption simultaneously. Signcryption can improve efficiency in processing time, broadband occupation, and key management. But Barbosa's scheme was pointed out to be vulnerable to malicious passive KGC attacks. Barreto et al.[18] proposed a certificateless signcryption scheme for bilinear pairs. In 2018, CAO et al.[19] proposed a signcryption scheme with privacy protection function. TA and PKG generated pseudonyms and keys of vehicles respectively. Schemes in literature[19] and literature[20] bilinear pairing operation adopted in the same way, which had low computational efficiency. At present, many scholars have studied signcryption technology[21-24], but no systematic scheme formed. Du et al.[25] put forward a certificateless signature scheme based on elliptic curve cryptosystemc but exist a replacement key attack. We improve Du et al's scheme, propose a certificateless signcryption scheme based on an elliptic curve, and the scheme is applied to the privacy protection of the IoV. The main contributions of this paper are as follows:

\section{Results}

- The ECC cryptography is used to construct pseudonyms, the traditional tamper-proof device(TPD) and password(PWD) are abandoned, the pseudonym is generated through the intermediate variable false identity and timestamp. So the scheme has strong privacy protection capability.

- Combining certificateless and signcryption theory, anonymous is introduced in the scheme. Key generation is related to RSUs, OBU, and KGC; the IBC algorithm is improved. Thus, the security of the key is enhanced.

- Computational cost decreased at least 18\% compared with other relevant schemes. The scheme satisfies the security of IND-CCA and EUF-CMA that makes the IoV system have forward security, anonymity, traceability, and can avoid replay attacks.

\section{Elliptic curve}

If $q$ is a large prime, it satisfies $q>=2^{160}, Z_{q}$ includes all solutions in the finite domain $F_{q}$ elliptic curve $E: y^{2}=x^{3}+a x+b \bmod q$, let $E\left(Z_{q}\right)$ denote the set of pairs $(x, y) \in\left(Z_{q} \times Z_{q}\right)$ satisfying the above equation along with a special value $O$. That is, $E\left(Z_{q}\right)=\left\{(x, y) \mid x, y \in Z_{q}, y^{2}=x^{3}+a x+b \bmod \right.$ $q\} \cup O$. The elements $E\left(Z_{q}\right)$ are called the points on the elliptic curve $E$, where $4 a^{3}+27 b^{2} \neq 0, O$ is called the point at infinity.

- Elliptic Curve Digital Signature Algorithm(ECDSA) is mainly used to create a digital signature for data and verify its authenticity without destroying 
security. Take a random integer $k$, Calculate the point $P=k G$, Calculate the number $r=x_{p} \bmod q$, where $r=x_{p}$ is the $x$ coordinate of $P$. Calculate $s=k^{-1}\left(z+r d_{A}\right) \bmod q, z$ is the hash truncation of message $M$.

- Elliptic curve discrete logarithmic problem (ECDLP), selecting additive cyclic group $G$ with order of the large prime $q, P$ is any generator of additive cyclic group $G$. It is know $P, a P \in G$, but it's unknown $a \in Z_{q}^{*}$, any probabilistic polynomial-time algorithm is difficult to compute the advantage in the $a$, looking for advantages in solution $\operatorname{Pr}[a \mid P, a P \in G]$ is considered negligible.

- Computational Diffie-Hellman $(\mathrm{CDH})$ problem, selecting additive cyclic group $G$ with order of the large prime $q, P$ is any generator of additive cyclic group $G$. It is known that $P, a P, b P \in G$, but it's unknown $a, b \in Z_{q}^{*}$, any probabilistic polynomial time algorithm is difficult to compute the advantage in the $a b P$, finding the solution $\operatorname{Pr}[a b P \mid P, a P, b P \in G]$ is considered negligible.

\section{System Overview}

In our scheme, the model of IoV is composed of vehicles, roadside units, key generator centers, and trusted authorities. The specific division of labor is as follows:

Onboard Unit (OBU): Vehicles equipped with OBU are intelligent and can exchange information and data with roadside units and other vehicles. Each vehicle periodically broadcasts information for safe driving. To ensure location privacy, each vehicle needs to use a pseudonym to replace its real identity to transmit information.

Roadside Units (RSUs): RSUs deploy along with urban roads. They are mainly composed of a wireless communication interface and local data preprocessing unit. The roadside units are deployed according to specific rules. Therefore, the vehicle can access the roadside units. All RSUs equipment should wire to the intelligent transportation information data center.

Trusted Authority (TA): TA is managed by the traffic management department and is mainly responsible for the identity registration and authentication of OBU. It is regarded as fully trusted in this scheme and is responsible for generating the false identity of the vehicle.

Key Generation Center (KGC): KGC is responsible for two-way communication with TA to generate partial public/private keys for legitimate OBU and RSUs.

The model as been shown in Figure 1. 


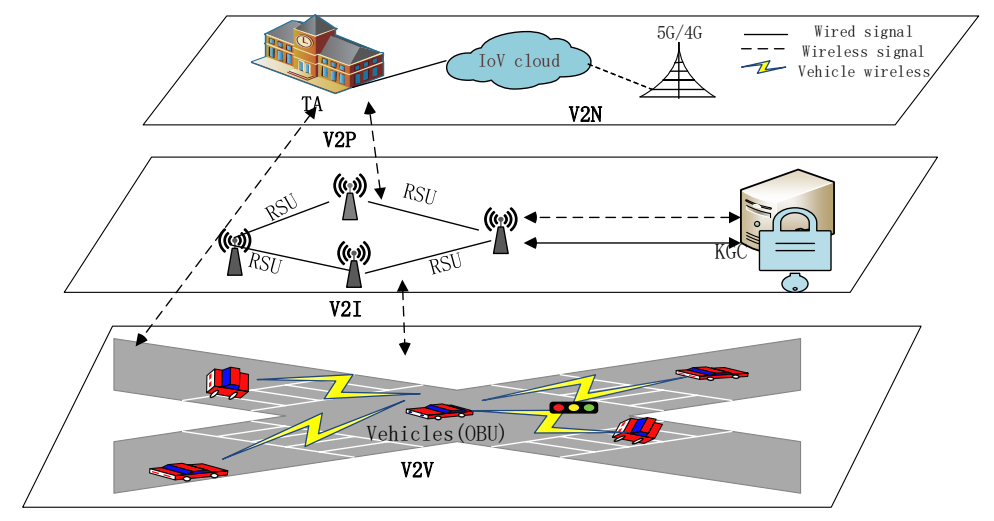

Fig. 1 System structure diagram of the IoV.

\subsection{Scheme}

The scheme is designed for IoV communication, avoids the problem of key escrow, the pseudonym mechanism is adopted to protect the real identity of both sides of the communication, ensures the confidentiality of the identity and the traceability of vehicles.

First, to eliminate the impact of replacing the public key in our scheme. The system-master-key is added to the pseudonym generation formula to enhance the difficulty of the attacker's forgery signature, making the $s$ cannot be bypassed. It can be seen that in the Du et al'scheme [25], part private key $S K_{i}$ was calculated by the system-master-key. The malicious signer cannot calculate the value of the system-master-key and $S K_{i}$ through technical means, but the public key of the certificateless signature scheme is not authenticated between the signer and the verifier, the malicious signer forges the signature by forging the secret value and bypassing the unknown system-master-key. Therefore, there is a key replacement attack. So, in our scheme, signcryption algorithm is introduced to ensure the confidentiality of transmission and improve transmission efficiency. Finally, the security of the scheme is proved in the standard model. The meaning of relevant symbols is shown in table1 and the flow chart of the algorithm is shown in figure 2. The algorithm steps are as follows: 
Table 1 Parameter Description Table.

\begin{tabular}{ll}
\hline line & Implications \\
\hline$G$ & Additive cyclic group of order q \\
$\mathrm{P}$ & Generator of group $\mathrm{G}$ \\
$\mathrm{s}$ & System Master Key \\
$Z_{q}^{*}$ & $Z_{q}^{*}=\{x: 0<x<q, g c d(x, q)=1\}$ \\
$H_{0}, H_{1}, H_{2}, H_{3}, H_{4}$ & five safe hash functions \\
$P_{j}, K_{j}, k_{j}$ & The identity of roadside unit j, public key $Y_{j}$, private key $y_{j}$ \\
$S_{i}$ & Partial private key \\
$r_{i}$ & KGC generate the secret value to generate public/private keys \\
$x_{i}$ & Secret value of the vehicle \\
$\xi_{i}$ & Secret value for the RSU \\
$P K_{i}, S K_{i}$ & Public key, private key for a vehicle \\
$R I D_{i}$ & List of true vehicle identities \\
$F_{i}$ & False identity of a vehicle \\
$F I D_{i}$ & Pseudonym of a vehicle \\
$T_{i}$ & Current timestamp of a vehicle \\
$\delta$ & Ciphertext between two vehicles \\
$Y, Y^{*}$ & Encryption key, Decryption key \\
$V_{A}, V_{B}$ & Vehicle of data sender, Vehicle of data receiver \\
$\mathbb{A}_{\mathrm{I}}, \mathbb{A}_{\mathrm{II}}$ & Type-I and Type-II adversaries \\
\hline
\end{tabular}

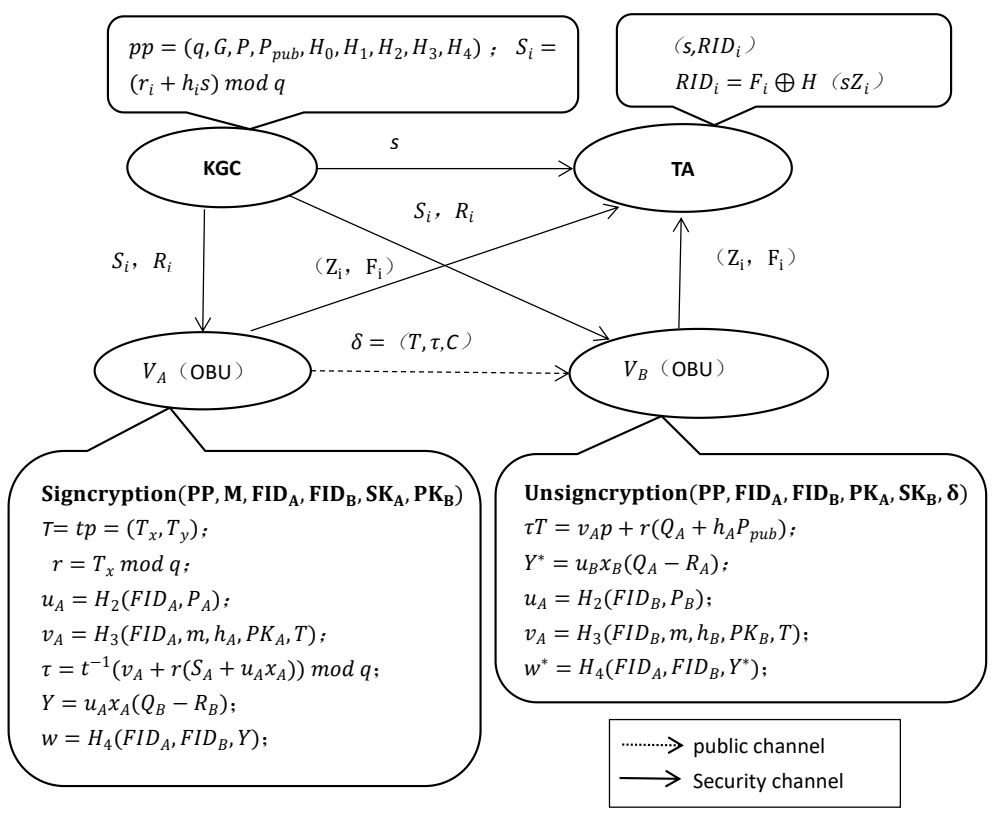

Fig. 2 The CLSC of our scheme. 


\subsection{Algorithm}

The certificateless signcryption scheme based on ECDSA, comprises five players: KGC, TA, RSU, the sender of vehicle $\left(V_{A}\right)$, and the receiver of vehicle $\left(V_{B}\right)$. OBU and RSU pass TA for two-way authentication[26]. We divide the whole scheme into six algorithms as follows:

\subsubsection{Initialization}

The KGC selects the five collision-resistant Hash functions:

$H_{0}:\{0,1\}^{*} \rightarrow Z_{q}^{*}$;

$H_{1}:\{0,1\}^{*} \times G \rightarrow Z_{q}^{*}$;

$H_{2}:\{0,1\}^{*} \times G \times G \rightarrow Z_{q}^{*}$;

$H_{3}:\{0,1\}^{*} \times Z_{q}^{*} \times G \times G \rightarrow Z_{q}^{*}$;

$H_{4}:\{0,1\}^{*} \times G \times G \rightarrow Z_{q}^{*}$.

The KGC secret saves system master key $s$ and transmits $s$ to TA, The TA saves $\left(s, R I D_{i}\right)$. The system public key is $P_{p u b}=s P$, then generates a common parameter $p p=\left(q, G, P, P_{p u b}, H_{0}, H_{1}, H_{2}, H_{3}, H_{4}\right)$.

\subsubsection{Registration}

The OBU executes the algorithm, OBU random selection $z_{i} \in Z_{i}^{*}$, calculates the negotiation key[27] $Z_{i}=z_{i} P$, generates false identity $F_{i}=R I D \oplus$ $H_{0}\left(z_{i} P_{p u b}\right)$, then sends $\left(Z_{i}, F_{i}\right)$ to TA. The algorithm is executed by TA, TA receives the message $\left(Z_{i}, F_{i}\right)$ from OBU. TA calculates $R I D_{i}=F_{i} \oplus H_{0}\left(s Z_{i}\right)$, queries whether the vehicle identity list containing $R I D_{i}$. If not, TA terminates the algorithm and determines it as an illegal OBU. RSU set identity as $P_{j}$, randomly selected $k_{i} \in Z_{q}^{*}$ as its private key, RSU calculate the negotiation key $K_{i}=k_{i} P$, the public key $K_{j}=k_{i} P_{p u b}$, and sents $\left(P_{j}, K_{i}\right)$ to TA, TA calcuates $\mathbb{K}_{j}=s K_{i}$ and forwards $\left(P_{j}, \mathbb{K}_{j}\right)$ to the legitimate OBU.

\subsubsection{Pseudonym generation}

The trusted organization no longer issues the public-key certificates(PKI) to vehicles but generates pseudonyms for them. In this scheme, the generation of a pseudonym consists of three parameters, including false identity of its own, RSU identity information, and timestamp, rather than the device password information.

When the vehicle enters the area responsible for the RSU, it receives $K_{j}$ from the RSU broadcast. The OBU checks the RSU's public key, if $K_{j} \notin$ $\left(P_{j}, \mathbb{K}_{j}\right)$, the RSU is illegal, the algorithm is not executed. Otherwise, the OBU obtains the current timestamp $T_{i}$ and the public key $K_{j}$ of the current $\mathrm{RSU}$, then selects the secret value $\xi_{i} \in Z_{q}^{*}$ for the RSU, the OBU calculates $F I D_{i 1}=F_{i} \oplus H_{0}\left(K_{j} \xi_{i} \| T_{i}\right), F I D_{i 2}=P_{j}$, the OBU sets the pseudonym of the vehicle $F I D_{i}=\left(F I D_{i 1}, F I D_{i 2}, T_{i}\right)$.

Through the above operations, TA indirectly judges the legitimacy of RSU. OBU generates the pseudonym through legal RSU, false identity of the vehicle, and the timestamp. 


\subsubsection{Key generation}

- Secret-Value: OBU chooses a random $x_{i} \in Z_{q}^{*}$ as the secret value.

- Partial-Private/Public-Key: KGC inputs the pseudonym of the vehicle $F I D_{i}$ and the parameter value $P P$, KGC chooses $r_{i} \in Z_{q}^{*}$ randomly, calculates partial public key $R_{i}=r_{i} P$, partial private key $S_{i}=\left(r_{i}+h_{i} s\right) \bmod q$, which $h_{i}=H_{1}\left(F I D_{i}, R_{i}\right)$. KGC via secure channel sends $\left(S_{i}, R_{i}\right)$ to OBU.

- Public-key-extract: OBU calculates $P_{i}=x_{i} P, u_{i}=H_{2}\left(F I D_{i}, P_{i}\right), Q_{i}=$ $R_{i}+u_{i} P_{i}$ then generates the public key is $P K_{i}=\left(R_{i}, Q_{i}\right)$.

- Private-key-extract: OBU checks whether the $S_{i} P=R_{i}+h_{i} P_{p u b}$ is established. If it established, it will be accepted. If not, it will be rejected. Generates the private key $S K_{i}=\left(S_{i}, x_{i}\right)$. Proof of correctness: $S_{i} P=$ $\left(r_{i}+h_{i} s\right) P=R_{i}+h_{i} P_{\text {pub }}$.

\subsubsection{Signcrption}

$V_{A}$ is the sender of OBU, $V_{B}$ is the receiver of OBU, $V_{A}$ takes message $M$, $F I D_{A}, F I D_{B}, P P, S K_{A}$ and $P K_{B}$ as input, and produces signcryptext $\delta$. The algorithm is as follows:

- $T=t P=\left(T_{x}, T_{y}\right), T_{x}, T_{y}$ are the $x$ coordinate value and $y$ coordinate value of point $T$.

- $\tau=t^{-1}\left(v_{A}+r\left(S_{A}+u_{A} x_{A}\right)\right) \bmod q$.

Where

$h_{A}=H_{1}\left(F I D_{A}, R_{A}\right)$;

$v_{A}=H_{3}\left(F I D_{A}, m, h_{A}, P K_{A}, T\right)$;

$r=T_{x} \bmod q$;

$u_{A}=H_{2}\left(F I D_{A}, P_{A}\right)$

- $C=M \oplus w$.

Where

$Y=u_{A} x_{A}\left(Q_{B}-R_{B}\right)$;

$w=H_{4}\left(F I D_{A}, F I D_{B}, Y\right)$.

$V_{A}$ send the $\delta=(T, \tau, C)$ to $V_{B}$.

\subsubsection{Unsigncryption}

$V_{B}$ takes $\delta, F I D_{A}, F I D_{B}, P P, S K_{B}$ and $P K_{A}$ as input, and returns massage $M$, if $\tau T=v_{A} P+r\left(Q_{A}+h_{A} P_{p u b}\right)$ is hold. $V_{B}$ performs the following steps:

- $w^{*}=H_{4}\left(F I D_{A}, F I D_{B}, Y^{*}\right)$;

- $Y^{*}=u_{B} x_{B}\left(Q_{A}-R_{A}\right)$;

- $u_{B}=H_{2}\left(F I D_{B}, P_{B}\right)$.

$V_{B}$ executes $M=C \oplus w^{*}$. 


\section{Correctness}

Only if the following two equations are true respectively, the scheme satisfies the correctness.

- Public verifiability. The message is signed by $V_{A}$, if the verification signature is valid, $V_{B}$ receives the message. Otherwise, if the signature is invalid, $V_{B}$ rejects the message.

$\tau T=t^{-1}\left(v_{A}+r\left(S_{A}+u_{A} x_{A}\right)\right) t p \bmod q$

$=\left(v_{A}+r\left(r_{A}+h_{A} s+u_{A} x_{A}\right)\right) P$

$=v_{A} P+r\left(R_{A}+u_{A} P_{A}+h_{A} P_{p u b}\right)$

$=v_{A} P+r\left(Q_{A}+h_{A} P_{p u b}\right)$.

- Consistency of encryption and decryption, if $Y^{*}=Y$ is true, $w^{*}=w$ must be true, $M=C \oplus w^{*}=M \oplus w \oplus w^{*}$ must be established.

$Y=u_{A} x_{A}\left(Q_{B}-R_{B}\right) ; Q_{B}-R_{B}=u_{B} P_{B}=u_{B} x_{B} P ; Y=u_{A} x_{A} u_{B} x_{B} P ;$

$Y^{*}=u_{B} x_{B}\left(Q_{A}-R_{A}\right) ; Q_{A}-R_{A}=u_{A} P_{A}=u_{A} x_{A} P ; Y^{*}=u_{B} x_{B} u_{A} x_{A} P ;$

$Y^{*}=Y ; w^{*}=w ;$

$M=C \oplus w^{*}=M \oplus w \oplus w^{*}=M \oplus w \oplus w$.

\section{Security proof}

To prove the security of our scheme, two types of adversaries are considered[28]. These security requirements are described via some games between an adversary $\left(\mathbb{A}_{I}\right.$ or $\left.\mathbb{A}_{I I}\right)$ and a challenger $\mathbb{C}$. Adversaries can be divided into two cases: one is that the adversary $\mathbb{A}_{I}$ is a malicious user attacker. The adversary $\mathbb{A}_{I}$ does not know the system master key $s$, but can replace the public key of any user; the second type of adversary $\mathbb{A}_{I I}$ is a malicious KGC attacker. This type of attacker knows the master key $s$ but cannot replace any public keys. In our CLSC scheme, the adversaries may access the following oracles:

- $H_{P K} \cdot F I D_{i}$ is entered as an identifier, a public-key $P K_{i}$ matching $F I D_{i}$ will be returned.

- $H_{d}$. FID $D_{i}$ is entered as an identifier, a partial-private key $S_{i}$ will be returned.

- $H_{\text {Replace.PK }} . F I D_{i}$ is entered as an identifier, a new public key $P K_{i}^{\prime}$ that can be used will replace the original public key $P K_{i}$.

- $H_{S K} . F I D_{i}$ is entered as an identifier, a private-key $S K_{i}$ matching $F I D_{i}$ will be returned, when the public-key is not replaced.

- $H_{\text {Signcrypt }}$. When there are a message $M$, identity of a sender $F I D_{A}$, and identity of a receiver $F I D_{B}$ as input, An available signcryption $\delta$ on $\mathrm{M}$ will be returned.

- $H_{U n s i g n c r y p t}$. When a signcryption $\delta$, identity of a sender $F I D_{A}$, and identity of a receiver $F I D_{B}$ are given, the message $M$ will be restored, when $\delta$ is available.

$\mathbb{A}_{I}$ can access all the above oracles, while $\mathbb{A}_{\text {II }}$ can access all of them except $H_{\text {Replace.PK }}$ and $H_{d}$, becuase $\mathbb{A}_{\text {II }}$ owns system-masterkey $s$, can forge partial-private key $\gamma, \mathbb{A}_{I}$ and $\mathbb{A}_{\text {II }}$ can suppose 
$H_{\mathrm{I}}=\left\{H_{P K}, H_{d}, H_{\text {Replace.PK }}, H_{S K}, H_{\text {Signcrypt }}, H_{\text {Unsigncrypt }}\right\}$ and $H_{\mathrm{II}}=$ $\left\{H_{P K}, H_{S K}, H_{\text {Signcrypt }}, H_{U n s i g n c r y p t}\right\}$, respectively.

We will prove this scheme from two aspects: confidentiality and unforgeability.

\subsection{Confidentiality}

This property is considered as the indistinguishability under chosen-ciphertext attack(IND-CCA). In this section, the security proof will be proved through some games between adversaries $\left(\mathbb{A}_{I}\right.$ or $\left.\mathbb{A}_{I I}\right)$ and a challenger $\mathbb{C}$.

Game 1 . the game interactions between an adversary $\mathbb{A}$ and a challenger $\mathbb{C}$ are as follows:

- Setup. $\mathbb{C}$ enters a security parameter $\lambda$, a common parameter $p p$ and $\alpha$ are generated, $\alpha$ is kept as secret.

- Phase 1 Queries. $\mathbb{A}_{I}$ sends bounded queries in polynomial time to the oracles in the $H_{\mathrm{I}}$, the $\mathbb{C}$ responses to these queries pass through these oracle models.

- Challenge. $\mathbb{A}_{\mathrm{I}}$ sends two equal length messages $m_{0}$ and $m_{1}$ to Challenger $\mathbb{C}$ with $F I D_{A}^{*}$ and $F I D_{B}^{*}$ as identifiers. $\mathbb{C}$ selects a bit $\gamma \in\{0,1\}$ randomly, implements Signcrption $\left(P P, M, F I D_{A}^{*}, F I D_{B}^{*}, S K_{A}^{*}, P K_{B}^{*}\right)$ then $\mathbb{C}$ sends $\delta$ to $\mathbb{A}_{I}$.

- Phase 2 Queries. $\mathbb{A}_{I}$ send bounded queries in polynomial time to the oracle $H_{\mathrm{I}}$, the $\mathbb{C}$ responses to these queries pass through these oracle models.

- Guess. $\mathbb{A}_{I}$ outputs a guess of $\gamma$ is $\gamma^{*}$.

It is said that $\mathbb{A}_{\mathrm{I}}$ wins Game 1 if $\gamma^{*}=\gamma$ and the following conditions established:

a. $\mathbb{A}_{\mathrm{I}}$ can't extract $S K_{A}^{*}$, at any point.

b. $\mathbb{A}_{\mathrm{I}}$ can't extract $S_{A}^{*}$, if $\mathbb{A}_{\mathrm{I}}$ has replaced $P K_{A}^{*}$ with $P K_{A}^{\prime}$ before accepting the challenge.

c. In Phase 2 queries, $\mathbb{A}_{\mathrm{I}}$ is unable to perform unsigncryption query on $\delta^{*}$ under $F I D_{A}^{*}$ or $F I D_{B}^{*}$, used to signcryption $M_{\gamma}, P K_{A}^{*}$ or $P K_{B}^{*}$ has been replaced after the challenge was issued.

Game 2. The game interactions between an adversary $\mathbb{A}$ and a challenger $\mathbb{C}$ : the challenge steps are the same as game 1 .

- Setup. $\mathbb{C}$ enters a security parameter $\lambda$, a common parameter $p p$ and $\alpha$ are generated. $\mathbb{C}$ sends parameter $p p$ and $\alpha$ to $\mathbb{A}_{\mathrm{II}}$.

- Phase 1 Queries. $\mathbb{A}_{I I}$ sends bounded queries in polynomial time to the oracles in the $H_{\mathrm{II}}$, the $\mathbb{C}$ responses to these queries pass through these oracle models.

- Challenge. $\mathbb{A}_{I I}$ sends two equal length messages $m_{0}$ and $m_{1}$ to Challenger $\mathbb{C}$ with $F I D_{A}^{*}$ and $F I D_{B}^{*}$ as identifiers. $\mathbb{C}$ selects a bit $\gamma \in\{0,1\}$ randomly, implements Signcryption $\left(P P, M, F I D_{A}^{*}, F I D_{B}^{*}, S K_{A}^{*}, P K_{B}^{*}\right)$ then $\mathbb{C}$ sends $\delta$ to $\mathbb{A}_{\text {II }}$. 
- Phase 2 Queries. $\mathbb{A}_{I I}$ sends bounded queries in polynomial time to the oracle $H_{\mathrm{II}}$, the $\mathbb{C}$ responses to these queries pass through these oracle models.

- Guess. $\mathbb{A}_{\text {II }}$ outputs a guess $\gamma^{*}$ of $\gamma$.

It is said that $\mathbb{A}_{\text {II }}$ wins Game 2 if $\gamma^{*}=\gamma$ and the following conditions hold:

a. $\mathbb{A}_{\mathrm{II}}$ can't extract $S K_{A}^{*}$ at any point. Because the secret value $x_{i}$ can't be obtained by $\mathbb{A}_{\mathrm{II}}$, adversary solves $x_{i}$ as ECDLP problem.

b. In Phase 2 queries, $\mathbb{A}_{I I}$ is unable to perform an unsigncryption query on $\delta^{*}$ under $F I D_{A}^{*}$ or $F I D_{B}^{*}$.

If this probability $\operatorname{Adv}(\mathbb{A})=2 *|\operatorname{Pr}[\mathbb{A}-1 / 2]|$ is negligible, we say the scheme is IND-CCA safe. We know that $\mathbb{A}_{I}$ can access to all of the oracles, while $\mathbb{A}_{I I}$ can access to all of them except $H_{\text {Replace.PK }}$ and $H_{d}$.

$\mathbb{A}_{\mathrm{I}}$ sends bounded queries in polynomial time to the oracles in the $H_{\mathrm{I}}$ make a signcryption query $H_{\text {Signcrypt }}$ but cannot win $\delta$ under FID $D_{A}^{*}$ and $F I D_{B}^{*}$. even if $\mathbb{A}_{\mathrm{I}}$ known key generation process $Q_{A}^{*}-R_{A}^{*}=u_{A}^{*} x_{A}^{*} P, Q_{B}^{*}-R_{B}^{*}=u_{B}^{*} x_{B}^{*} P$, $Y=u_{B}^{*} x_{B}^{*} u_{A}^{*} x_{A}^{*} P$. Solving $Y$ is still difficult, it is the $\mathrm{CDH}$ problem.

$\mathbb{A}_{\text {II }}$ sends bounded queries in polynomial time to the oracles in the $H_{\text {II }}$ make an public-key query $H_{P K}$, but $H_{\text {II }}$ cannot obtain $x_{i}^{*}$, thus cannot obtain $P K_{i}$. Solving $x_{i}^{*}$ is ECDLP problem.

$A d v(\mathbb{A})$ the probability of winning game 1 and game 2 is negligible.

\subsection{Unforgeability}

This property is considered as the existential unforgeability against chosen message attack(EUF-CMA). In this section, the security proof will be proved through some games between adversaries $\left(\mathbb{A}_{\mathrm{I}}\right.$ or $\left.\mathbb{A}_{\text {II }}\right)$ and a challenger $\mathbb{C}$.

Game 3. The game interactions between an adversary $\mathbb{A}$ and a challenger $\mathbb{C}$ are as follows:

- Setup. $\mathbb{C}$ enters a security parameter $\lambda$, a common parameter $p p$ and $\alpha$ are generated, $\alpha$ is kept as secret.

- Phase 1 Queries. $\mathbb{A}_{I}$ sends bounded queries in polynomial time to the oracles in the $H_{\mathrm{I}}$, the $\mathbb{C}$ responses to these queries pass through these oracle models.

- Forgery. $\mathbb{A}_{I}$ forges the message $M^{*}$ and signcryption $\delta^{*}=\left(T^{*}, \tau^{*}, C^{*}\right)$ from the send $V_{A}^{*}$ to the receiver $V_{B}^{*}$.

$\mathbb{A}_{\mathrm{I}}$ wins Game 3 if Unsigncrption $\left(P P, F I D_{A}^{*}, F I D_{B}^{*}, P K_{A}^{*}, S K_{B}^{*}, \delta\right)$ output $M^{*}$ and the following conditions hold:

a. $\mathbb{A}_{\mathrm{I}}$ can't extract $S K_{A}^{*}$ at any point.

b. $\mathbb{A}_{\mathrm{I}}$ can't extract $S K_{i}^{*}$ for any pseudonym $F I D_{i}$, if $P K_{i}^{*}$ has been replaced.

c. $\mathbb{A}_{\mathrm{I}}$ cannot extract $x_{A}^{*}$.

d. $\mathbb{A}_{\mathrm{I}}$ can't make a signcryption query on $M^{*}$ under $F I D_{A}^{*}$ and $F I D_{B}^{*}$.

Game 4 . The game interactions between an adversary $\mathbb{A}$ and a challenger $\mathbb{C}$ : the challenge steps are the same as game 3 . 
- Setup. $\mathbb{C}$ enters a security parameter $\lambda$, a common parameter $p p$ and $\alpha$ are generated. $\mathbb{C}$ sends parameter $p p$ and $\alpha$ to $\mathbb{A}_{\mathrm{II}}$.

- Queries. $\mathbb{A}_{I I}$ sends bounded queries in polynomial time to the oracles in the $H_{\mathrm{II}}$, the $\mathbb{C}$ responses to these queries pass through these oracle models.

- Forgery. $\mathbb{A}_{I I}$ creates a forged message $m^{*}$ or signcryption $\delta^{*}=\left(T^{*}, \tau^{*}, C^{*}\right)$ from the send $V_{A}^{*}$ to the receiver $V_{B}^{*}$.

It is said that $\mathbb{A}_{\mathrm{II}}$ wins Game 4 if the output of Unsigncrption $\left(P P, F I D_{A}^{*}, F I D_{B}^{*}, P K_{A}^{*}, S K_{B}^{*}, \delta\right)$ is $M^{*}$ and the following conditions hold:

a. $\mathbb{A}_{\text {II }}$ can't extract $S K_{A}^{*}$ at any point.

b. $\mathbb{A}_{I I}$ can't make a signcryption query on $M^{*}$ under $F I D_{A}^{*}$ and $F I D_{B}^{*}$.

If $\mathbb{A}_{\mathrm{I}}$ or $\mathbb{A}_{\mathrm{II}}$ winning game 3 and game 4 is negligible $\left(\operatorname{Adv} \operatorname{Sig}_{\epsilon, A}^{C M A}(k) \leq\right.$ $n e g l(k))$, we say the scheme is EUF-CMA safe. Note that $\mathbb{A}_{\mathrm{I}}$ has access to all of the mentioned oracles, while $\mathbb{A}_{\mathrm{II}}$ has access to all of them except $H_{\text {Replace.PK }}$ and $H_{d}$.

$\mathbb{A}_{\mathrm{I}}$ executes public key replacement queries from $H_{\text {Replace.PK }}$, can replace the public key with $P K_{A}^{\prime}=\left(R_{A}, Q_{A}^{\prime}\right), P K_{B}^{\prime}=\left(R_{B}, Q_{B}^{\prime}\right)$, signcryption queries from $H_{\text {Signcrypt }}$ and unsigncryption queries from $H_{U \text { usigncryption }}, \mathbb{A}_{\mathrm{I}}$ random selects $t^{*} \in Z_{q}^{*}, x_{A}^{*} \in Z_{q}^{*}, x_{B}^{*} \in Z_{q}^{*}$ computes $T^{*}=t^{*} P=\left(T_{x}, T_{y}\right), r^{*}=$ $T_{x} \bmod q, v_{A}^{*}=H_{3}\left(F I D_{A}^{*}, m, h_{A}^{*}, P K_{A}^{\prime}, T\right)$, forged $Q_{A}^{\prime}=x_{A}^{*} P-h_{A}^{*} P_{p u b}$, $Q_{B}^{\prime}=x_{B}^{*} P-h_{B}^{*} P_{p u b}$; to signcrypt the message $m^{*}$. Then forged signcryption $\delta^{*}=\left(T^{*}, \tau^{*}, C^{*}\right), V_{B}$ receives $\delta^{*}$ and conducts feasibility verification:

$$
\begin{aligned}
& \tau^{*} T^{*}=t_{A}^{*-1}\left(v_{A}^{*}+r^{*} x_{A}^{*}\right) t_{A}^{*} P=\left(v_{A}^{*}+r^{*} x_{A}^{*}\right) P=v^{*} P+r^{*}\left(Q_{A}^{\prime}+h_{A}^{*} P_{p u b}\right) ; \\
& Y^{\prime}=u_{A}^{*} x_{A}^{*}\left(Q_{B}^{\prime}-R_{B}\right)=u_{A} x_{A}\left(x_{B}^{*} P-h_{B}^{*} P_{p u b}-R_{B}\right) ; \\
& Y^{*}=u_{B}^{*} x_{B}^{*}\left(Q_{A}^{\prime}-R_{A}\right)=u_{B} x_{B}\left(x_{A}^{*} P-h_{A}^{*} P_{p u b}-R_{A}\right) ;
\end{aligned}
$$

Because $Y^{*} \neq Y^{\prime} ; w^{*} \neq w^{\prime}$, so $m^{\prime}=C^{*} \oplus w^{*}=m^{*} \oplus w \oplus w^{*} \neq m^{*}$. $\mathbb{A}_{\mathrm{I}}$ challenge failure.

$\mathbb{A}_{\text {II }}$ cannot execute query partial-private key from $H_{d}$; thus, forged $\gamma$ replace $x_{A}^{*}$, select $t^{\prime} \in Z_{q}^{*}$, forged $\delta^{*}=\left(T^{*}, \tau^{*}, C^{*}\right) ; T^{*}=t^{\prime} P ; \tau^{*}=t^{\prime-1}\left(v_{A}+r\left(S_{A}+\right.\right.$ $\left.\left.u_{A}^{\prime} \gamma\right)\right) \bmod q$; which $P_{A}^{\prime}=\gamma P ; u_{A}^{\prime}=H_{2}\left(F I D_{A}, P_{A}^{\prime}\right) ; V_{B}$ get $\delta^{*}$ then feasibility verification.

$$
\begin{aligned}
& \tau^{*} T^{*}=\left(t^{\prime-1}\left(v+r\left(S_{A}+u_{A}^{\prime} \gamma\right)\right)\right) t^{\prime} P \bmod q \\
& =\left(v^{\prime}+r\left(r_{A}+h_{A} s+u_{A}^{\prime} \gamma\right)\right) P \\
& =v^{\prime} P+r\left(R_{A}+h_{A} P_{p u b}+u_{A}^{\prime} P_{A}\right)
\end{aligned}
$$

for $\mathbb{A}_{\text {II }}$ cannot replace any public keys, thus $Q_{A} \neq R_{A}+h_{A} P_{A} ; \tau^{*} T^{*} \neq$ $v P+r\left(Q_{A}+h_{A} P_{p u b}\right)$. We know if the equation does not hold, output INVALID, $V_{B}$ discard ciphertext.

$A d v(\mathbb{A})$ the probability of winning game 3 and game 4 is negligible.

\section{Performance evaluation}

This section analyzes the present scheme from security, computational cost, and communication cost. It is compared with other relevant schemes [30-35]. 
Table 2 Run time of the different encryption operations.

\begin{tabular}{llll}
\hline Symbol & operation & parameter & Runtimec \\
\hline & & & \\
$T_{e m}$ & Elliptic curve point multiplication & $x \cdot P\left(P \in G, x \in z_{q}^{*}\right)$ & $0.341 \mathrm{~ms}$ \\
$T_{i n}$ & Inverse mode & $t^{-1} \bmod q\left(t \in z_{q}^{*}, q \in z_{q}^{*}\right)$ & $0.029 \mathrm{~ms}$ \\
$T_{e a}$ & Elliptic curve point plus & $P+Q(P \in G, Q \in G)$ & $0.002 \mathrm{~ms}$ \\
$T_{b p}$ & Time required for the bilinear pairing & $e(\bar{S}, \bar{T})\left(\bar{S} \in G_{1}, \bar{T} \in G_{1}\right)$ & $4.669 \mathrm{~ms}$ \\
$T_{p m}$ & Pairing multiplication operation & $\bar{x} \cdot \bar{P}\left(\bar{x} \in z_{q}^{*}, \bar{P} \in G\right)$ & $0.788 \mathrm{~ms}$ \\
$T_{p a}$ & Pairing addition & $\bar{S}+\bar{T}\left(\bar{S} \in G_{1}, \bar{S} \in G_{1}\right)$ & $0.002 \mathrm{~ms}$ \\
$T_{m t p}$ & MapToPoint hash function & $H_{1}: 0,1^{*} \rightarrow G_{1}$ & $0.145 \mathrm{~ms}$ \\
$T_{e}$ & Modular exponentiation & $g^{*} \bmod n$ & $1.915 \mathrm{~ms}$ \\
\hline
\end{tabular}

These schemes selected for comparison are certificateless signcryption and can be applied to the IoV.

The computational cost mainly depends on the amount of computation of signcryption algorithm and verification calculation for decryption. It can be measured by the number of execution times of statistical elliptic curve scalar multiplication, elliptic curve scalar addition, bilinear pairing, and mapping to point operation. The computational cost of XOR operation on $Z_{q}^{*}$ is small, so that no comparison. The operation results are in table 2. The experimental system environment:CPU:

Intel core i7-6700@3.40GHz; RAM:8GB;

OS:Ubuntu16.04;

Library: MIRACL, a public C++cryptographic library;

[https://github.com/miracl/MIRACL/archive/master.zip].

Communication cost is measured by the length of a single ciphertext. In the bilinear pairing operation scheme, the length of $\left|G_{1}\right|$ is 1024 bits, the length of $\left|G_{2}\right|$ is the same as $\left|G_{1}\right|$. To provide the same level of security scheme, for the scheme based on the elliptic curve, $q$ is the prime number, the length of $\left|Z_{q}^{*}\right|$ is 160 bits. The additive cyclic group with $q$ order generation for point $P$ on a nonsingular elliptic curve is $G$, the length of $|G|$ is 320 bits. Our scheme is designed according to the certificateless signcryption model, relies on ECDSA, and depends on the difficulty of pseudonym generation. This section will compare and analyze the security of the algorithm with similar schemes. The result is in table 3 . The superiority of this scheme is illustrated by comparing the calculation cost and communication cost of a single ciphertext, which is statistically analyzed in table 4 . Under the same operating environment, our scheme costs $1.397 \mathrm{~ms}$, Kasyoka et al's scheme[30] costs $1.705 \mathrm{~ms}$, Karati et al's scheme[31] based no pairing costs $2.424 \mathrm{~ms}$, Karati et al's scheme[32] based on bilinear pairing costs $18.913 \mathrm{~ms}$, He et al's[33] costs $2.05 \mathrm{~ms}$ and Seo et al's[35] costs $3.41 \mathrm{~ms}$. Compared with the other schemes [30-33] and [35]. Our scheme in this paper increases by $18.06 \%, 42.37 \%, 92.61 \%, 31.85 \%$ and $59.03 \%$ respectively. 
Table 3 safety comparison.

\begin{tabular}{lllll}
\hline Scheme & Confidentiality & Unforgeability & Forward security & Anonymous \\
\hline$[30]$ & false & true & false & false \\
{$[31]$} & false & false & false & false \\
{$[32]$} & true & false & false & false \\
{$[33]$} & false & true & false & true \\
{$[34]$} & false & true & false & false \\
{$[35]$} & true & true & false & false \\
Our-CLSC & true & true & true & true \\
\hline
\end{tabular}

Table 4 performance comparison of different signcryption schemes.

\begin{tabular}{llllllll}
\hline \multirow{2}{*}{ Scheme } & \multicolumn{3}{c}{ Calculate cost } & & \multicolumn{2}{c}{ Communication cost } \\
\cline { 2 - 3 } \cline { 6 - 8 } & Signcryption & Unsigncryption & Runtime & & signcryptext & Length \\
\hline$[30]$ & $2 T_{e m}$ & $3 T_{e m}$ & 1.705 & & $3\left|Z_{q}^{*}\right|$ & 480 \\
{$[31]$} & $3 T_{e m}+2 T_{e a}+T_{i n}$ & $4 T_{e m}+2 T_{e a}$ & 2.424 & & $2\left|Z_{q}^{*}\right|+|G|$ & 640 & $\mathrm{c}$ \\
{$[32]$} & $3 T_{e}$ & $2 T_{e}+2 T_{b p}$ & 18.913 & & $4\left|G_{1}\right|+\left|Z_{q}^{*}\right|$ & 4256 & \\
{$[33]$} & $3 T_{e m}$ & $3 T_{e m}+2 T_{e a}$ & 2.05 & & $3|G|+\left|Z_{q}^{*}\right|$ & 1120 \\
{$[35]$} & $3 T_{e m}$ & $7 T_{e m}$ & 3.41 & & $3\left|Z_{q}^{*}\right|$ & 480 \\
Our-CLSC & $T_{i n}+T_{e m}$ & $2 T_{e a}+3 T_{e m}$ & 1.397 & & $2\left|Z_{q}^{*}\right|+|G|$ & 640 \\
\hline
\end{tabular}

In the comparative analysis of communication cost, the length of a single ciphertext is used as the unit of comparison. The length of the ciphertext in our scheme is 640bits, which is slightly higher than Kasyoka et al's[30] and Seo et al's[35], lower than Karati et al's bilinear pairing scheme[32] and He et al's[33], the same as no pairing scheme of Karati et al.[31].

\section{Security analysis}

\subsection{Forward security}

If the system master key $s$ was leaked, it is calculated due to the difficulty of ECDLP, calculates $r_{i}, x_{i}$ still difficult, $\left(P K_{i}, S K_{i}\right)$ remains unknown. Therefore, it is guaranteed that the past signcryption information will not be disclosed, because of the randomness of $r_{i}, x_{i}$. When the system master key is leaked, the new values will immediately replace for them. The key update is realized, these actions further confirm the security of the communication[29].

\subsection{Traceability}

The ciphertext should contain relevant information about the identity of the vehicle. In the scheme, the TA can calculate $R I D_{i}=F_{i} \oplus H_{0}\left(s Z_{i}\right)$ by using the system master key $s$, which queries whether $R I D_{i}$ is in the vehicle identity list. It seems that only the trusted authority TA can track the vehicle according to this relevant information. In addition, the Internet of vehicles requires an extremely high real-time nature, and the ciphertext contains timestamp information, which can also prevent replay attacks. Because ciphertext 
$C=M \oplus w ; w=H_{4}\left(F I D_{A}, F I D_{B}, Y\right)$, here we can use the pseudonym of the vehicle $F I D_{i}=\left(F I D_{i 1}, F I D_{i 2}, T_{i}\right)$ making the ciphertext contains timestamp information.

\subsection{Anonymous}

Pseudonyms are used in V2V and V2I communications to protect the true identity of the vehicle. The pseudonym of the vehicle consists of three parts $F I D_{i}=\left(F I D_{i 1}, F I D_{i 2}, T_{i}\right)$ where $F I D_{i 1}$ is generated by the false identity $F_{i}$ of the vehicle $F I D_{i 1}=F_{i} \oplus H_{0}\left(K_{j} \xi_{i} T_{i}\right), F_{i}=R I D_{i} \oplus H_{0}\left(z_{i} P_{p u b}\right), F I D_{i 2}=P_{j}$, $T_{i}$ is the timestamp, to ensure the anonymity of the vehicle, it is necessary to protect the identity information $R I D_{i}$ of the vehicle when the pseudonym information is disclosed. According to the irreversibility of a hash function and the difficulty of ECDLP, the attacker cannot calculate $z_{i}, \xi_{i}$ and $k_{i}$ in polynomial time, so he cannot obtain the $R I D_{i}$ of the vehicle. In addition, vehicles carry different pseudonyms in different RSU communication ranges and different timestamps, that is, the vehicle pseudonym information changes with position and time, which makes the generation process of a pseudonym is the trapdoor one-way function.

\section{Conclusion}

In this paper, we constructed a reliable certificateless signcryption scheme without bilinear, where a pseudonym mechanism was also designed to protect the privacy of vehicles. We use certificateless signcryption technology to implement the scheme, which can secure vehicular communications with a low computational overhead. Performance analysis demonstrates that our proposed scheme reduces computational cost and communication cost compared with other related schemes. Security proves and analysis shows that our proposed scheme can avoid replacement public-key attacks, satisfy the security of IND-CCA and EUF-CMA, and other security requirements including perfect forward secrecy, anonymity, traceability, and resistance of replay attacks.

Supplementary information. c If your article has accompanying supplementary file/s please state so here.

Authors reporting data from electrophoretic gels and blots should supply the full unprocessed scans for key as part of their Supplementary information. This may be requested by the editorial team/s if it is missing.

Please refer to Journal-level guidance for any specific requirements.

\section{Declarations}

- Authors' contributions. This paper is completed by all authors. Beibei.C. is responsible for proposing the idea of the paper, Beibei.C. checks whether the idea is feasible and gives some suggestions to improve this idea. Beibei.C. 
is responsible for writing this paper, and the security analysis is completed by Beibei.C. Lu. Wei. is responsible for the performance analysis and comparison. Finally, the language of the paper is improved by Wei.H.

- Funding. In part by the funding project for top talent cultivation in Colleges and Universities of Anhui Province under Grant gxgnfx2020178, in part by the Natural Science Foundation of Anhui Province under Grant KJ2018A0944.

- acknowledgments. The authors thank the Associate Editor and the anonymous reviewers for their useful comments and suggestions which helped us improve the quality and presentation of this paper.

- Conflict of interest. The authors declare no conflict of interest.

If any of the sections are not relevant to your manuscript, please include the heading and write 'Not applicable' for that section.

\section{References}

[1] Cui J, Zhang X, Zhong H, et al. Extensible Conditional Privacy Protection Authentication Scheme for Secure Vehicular Networks in a MultiCloud Environment [J]. IEEE Transactions on Information Forensics and Security, 2020, 15(1): 1654-1667.

[2] Song C, Zhang M Y, Peng W P, et al. Research on anonymous authentication scheme in VANET[J]. Journal of Chinese Computer Systems,2018, 39(5): 899-903.

[3] Kamat P, Baliga A, Trappe W. An identity-based security framework For VANETs $[\mathrm{C}] / /$ International Workshop on Vehicular Ad Hoc Networks. ACM, 2006.

[4] A.Shamir. Identity-based cryptosystems and signature schemes. In Proceedings of CRYPTO 84 on Advances in Proceedings of CRYPTO 84 on Advances in cryptology 1985.

[5] Zhang J, Cui J, Zhong H, et al. PA-CRT: Chinese Remainder Theorem Based Conditional Privacy-preserving Authentication Scheme in Vehicular Ad-hoc Networks[J]. IEEE Transactions on Dependable and Secure Computing, 2019:1-1.

[6] Raya M, Hubaux J P. Securing vehicular ad hoc networks [J]. Journal of Computer Security, 2007, 15(1): 39-68.

[7] Tzeng S F, Horng S J, Li T, et al. Enhancing security and privacy for identity-based batch verification scheme in VANETs[J]. IEEE Transactions on Vehicular Technology, 2017, 66(4): 3235-3248. 
[8] Al-Riyami S, Paterson K G. Certificateless public key cryptography [C] //International conference on the theory and application of cryptology and information security. Springer, Berlin, Heidelberg, 2003: 452-473.

[9] Liu J K, Au M H, Susilo W. Self-generated-certificate public key cryptography and certificateless signature/encryption scheme in the standard model $[\mathrm{C}] / /$ Proceedings of the 2nd ACM symposium on Information, computer and communications security. Springer, Berlin, Heidelberg, 2007: 273-283.

[10] Shim K A. A new certificateless signature scheme provably secure in the standard model[J].IEEE Systems Journal, 2018, 13(2): 1421-1430.

[11] Yang W, Wang S, Wu W, et al. Top-Level secure certificateless signature against malicious-but-passive KGC[J]. IEEE Access, 2019, 7: 112870112878 .

[12] Thumbur G, Rao G S, Reddy P V, et al. Efficient pairing-free certificateless signature scheme for secure communication in resource-constrained devices[J]. IEEE Communications Letters, 2020, 24(8): 1641-1645.

[13] Mei Q,Xiong Hu, Chen J H, et al. Efficient certificateless aggregate signature with conditional privacy preservation in IoV[J]. IEEE System Journal, 2020: 1-12.

[14] Ali I, Lawrence T, Li F G. An efficient identity-based signature scheme without bilinear pairing for vehicle-to-vehicle communication in VANETs[J]. Journal of Systems Architecture, 2020, 103: 101692-101705.

[15] Barbosa M, Farshim P. Certificateless signcryption. In ACM Symposium on Information, Computer and Communications Security-ASIACCS 2008: 369-372.

[16] Zheng Y. Digital signcryption or how to achieve cost(signature \& encryption)iicost (signature)+cost (encryption) [C]//Annual international cryptology conference. Springer, Berlin, Heidelberg, 1997: 165-179.

[17] Cui J, Wei L, Zhong H, et al. Edge Computing in VANETs-An Efficient and Privacy-Preserving Cooperative Downloading Scheme[J]. IEEE Journal on Selected Areas in Communications, 2020, 38(6):1191-1204.

[18] Barreto P, Deusajute A, Cruz E, et al. Toward efficient certiicateless signcryption from (and without) bilinear pairings [EB/OL].(2008)

[19] Suzhen CAO,Xiaoli LANG, Xiangzhen LIU, et al. New Heterogeneous Signcryption Scheme under 5G Network[J].Netinfo Security, 2018, 18(11): 33-39. 
[20] Li FG, Masaaki S, Tsuyoshi T. Certificateless hybrid signcryption. Mathematical and Computer Modelling 2013, 57(3-4):324-343. [doi: 10.1016/j.mcm.2012.06.011]

[21] Liu Z, Hu Y, Zhang X, et al. Certificateless signcryption scheme in the standard model[J]. Information Sciences, 2010, 180(3): 452-464.

[22] Zhou C, Zhou W, Dong X. Provable certificateless generalized signcryption scheme [J]. Designs, Codes and cryptography, 2014, 71(2):331-346.

[23] Luo M, Tu M, Xu J. A security communication model based on certificateless online/offline signcryption for Internet of Things. Security \& Communication Networks, 2013,7(10):1560-1569.

[24] Yu HF, Yang B. Provably secure certificateless hybrid signcryption. Chinese Journal of Computers, 2015, 38(4):804-813 (in Chinese with English abstract).

[25] Du H, Wen Q, Zhang S, et al. A new provably secure certificateless signature for Internet of Things[J]. Ad Hoc Networks, 2020, 100: 102074102084.

[26] Wei L, Cui J, Zhong H, et al. Proven Secure Tree-based Authenticated Key Agreement for Securing V2V and V2I Communications in VANETs[J]. IEEE Transactions on Mobile Computing, 2021, PP(99):1-1.

[27] J Zhang, Zhong H, J Cui, et al. SMAKA: Secure Many-to-Many Authentication and Key Agreement Scheme for Vehicular Networks[J]. IEEE Transactions on Information Forensics and Security, 2020, PP(99):1-1.

[28] Parvin R, Willy S, Mohammad D. Efficient Certificateless Signcryption in the Standard Model: Revisiting Luo and Wan's Scheme from Wireless Personal Communications (2018)[J]. The Computer Journal, 2019(8):8.

[29] Wei L, Cui J, Xu Y, et al. Secure and Lightweight Conditional PrivacyPreserving Authentication for Securing Traffic Emergency Messages in VANETs[J]. IEEE Transactions on Information Forensics and Security, 2020, $\operatorname{PP}(99): 1-1$.

[30] Kasyoka P, Kimwele M, Angolo S M. Cryptanalysis of a pairing-free certificateless signcryption scheme[J], ICT Express 7, 2021, 200-204.

[31] Karati A, Fan C I, Huang J J. An efficient pairing-free certificateless signcryption without secure channel communication during secret key issuance[J]. Procedia Computer Science, 2020, 171: 110-119. 
[32] Karati A, Fan C I, Hsu R, et al. Provably secure and generalized signcryption with public verifiability for secure data transmission between resource-constrained IoT devices[J]. IEEE Internet of Things Journal: 2019, 6(6): 10431-10440.

[33] He D, Zeadally S, Xu B, et al. An efficient identity-based conditional privacy-preserving authentication scheme for vehicular ad hoc networks[J]. IEEE Transactions on Information Forensics and Security, 2015, 10(12): 2681-2691.

[34] Jia X, He D, Liu Q, et al. An Efficient Provably-Secure Certificateless Signature Scheme for Internet-of-Things Deployment[J]. Ad Hoc Networks, 2018, 71(MAR.):78-87.

[35] Seo S H, Won J, Bertino E. pCLSC-TKEM: a Pairing-free Certificateless Signcryption-tag Key Encapsulation Mechanism for a PrivacyPreserving IoT[J]. Transactions on Data Privacy, 2016, 9(2): 101-130. 